FACTA UNIVERSITATIS (NIŠ)

Ser. Math. Inform. Vol. 35, No 5 (2020), 1239-1250

https://doi.org/10.22190/FUMI2005239E

\title{
BANACH FIXED POINT THEOREM ON ORTHOGONAL CONE METRIC SPACES
}

\author{
Zeinab Eivazi Damirchi Darsi Olia, Madjid Eshaghi Gordji \\ and Davood Ebrahimi Bagha
}

(C) 2020 by University of Niš, Serbia | Creative Commons Licence: CC BY-NC-ND

\begin{abstract}
In this paper, we introduce new concept of orthogonal cone metric spaces. We establish new versions of fixed point theorems in incomplete orthogonal cone metric spaces. As an application, we show the existence and uniqueness of solution of the periodic boundry value problem.

Keywords: Orthogonal set; Fixed point; Orthogonal cone metric space; Differential equation; Solution.
\end{abstract}

\section{Introduction}

The concept of standard metric spaces is a fundamental tool in topology, functional analysis and nonlinear analysis. This structure has attracted a considerable attention from mathematicians because of the development of the fixed point theory in standard metric spaces.

In recent years, several generalizations of standard metric spaces have appeared. Huang and Zhang [8] have introduced the concept of cone metric space by replacing the set of real numbers by an ordered Banach space and proved many fixed point theorems of contractive type mappings in cone metric space. In 2010, W.S.Du [2] has shown that many results in fixed point theory on cone metric spaces are equivalent to ordinary metric spaces. Subsequently, many authors in [2, 7, 9] have generalized the results of Huang and Zhang [8].

Huang and Zhang [8] considered the concept of cone metric spaces as follows:

Definition 1.1. [8] Let $E$ always be a real Banach space and $P$ a subset of $E . P$ is called a cone if and only if:

1. $P$ is closed, nonempty, and $P \neq\{0\}$;

Received June 30, 2019; accepted March 09, 2020

2020 Mathematics Subject Classification. Primary 47H10; Secondary 54H25 
2. $a, b \in \mathbb{R}, a, b \geq 0, x, y \in P$ then $a x+b y \in P$;

3. $x \in P$ and $-x \in P$ then $x=0$.

Given a cone $P \subset E$, we define a partial ordering $\leqslant$ with respect to $P$ by $x \leqslant y$ if and only if $y-x \in P$. We shall write $x<y$ to indicate that $x \leqslant y$ but $x \neq y$, while $x \ll y$ will stand for $y-x \in$ int $P$, int $P$ denotes the interior of $P$. The cone $P$ is called normal if there is a number $K>0$ such that for all $x, y \in E$,

$$
0 \leq x \leq y \Longrightarrow\|x\| \leq K\|y\|
$$

The least positive number satisfying above is called the normal constant of $P$.

The cone $P$ is called regular if every increasing sequence which is bounded from above is convergent. That is, if $\left\{x_{n}\right\}$ is sequence such that

$$
x_{1} \leq x_{2} \leq \cdots \leq x_{n} \leq \cdots \leq y,
$$

for some $y \in E$, then there exists $x \in E$ such that $\left\|x_{n}-x\right\| \rightarrow 0$ as $n \longrightarrow \infty$. Equivalently the cone $P$ is regular if and only if every decreasing sequence which is bounded from below is convergent. It is well known that a regular cone is a normal cone.

Definition 1.2. [8] Let $X$ be a nonempty set. Suppose the mapping $d: X \times X \rightarrow E$ satisfies

- (d1) $0<d(x, y)$ for all $x, y \in X$ and $d(x, y)=0$ if and only if $x=y$;

- (d2) $d(x, y)=d(y, x)$ for all $x, y \in X$;

- (d3) $d(x, y) \leq d(x, z)+d(y, z)$ for all $x, y, z \in X$.

Then $d$ is called a cone metric on $X$, and $(X, d)$ is called a cone metric space.

It is obvious that cone metric spaces generalize metric spaces.

Example 1.1. Let $E=\mathbb{R}^{2}, P=\{(x, y) \in E \mid x, y \geq 0\} \subset \mathbb{R}^{2}, X=\mathbb{R}$ and $d: X \times X \rightarrow E$ such that $d(x, y)=(|x-y|, \alpha|x-y|)$, where $\alpha \geq 0$ is a constant. Then $(X, d)$ is a cone metic space.

Definition 1.3. $\quad[8]$ Let $(X, d)$ be a cone metric space. Let $\left\{x_{n}\right\}$ be a sequence in $X$ and $x \in X$. If for every $c \in E$ with $0 \ll c$ there is $N$ such that for all $n>N$, $d\left(x_{n}, x\right) \ll c$, then $\left\{x_{n}\right\}$ is said to be convergent and $\left\{x_{n}\right\}$ converges to $x$ and $x$ is the limit of $\left\{x_{n}\right\}$. We denote this by

$$
\lim _{n \rightarrow \infty} x_{n}=x \quad \text { or } x_{n} \rightarrow x \quad(n \rightarrow \infty) .
$$

Definition 1.4. [8] Let $(X, d)$ be a cone metric space, $\left\{x_{n}\right\}$ be a sequence in $X$. If for any $c \in E$ with $0 \ll c$, there is $N$ such that for all $n, m>N, d\left(x_{n}, x_{m}\right) \ll c$, then $\left\{x_{n}\right\}$ is called a Cauchy sequence in $X$. 
Definition 1.5. [8] Let $(X, d)$ be a cone metric space if every Cauchy sequence is convergent in $X$, then $X$ is called a complete cone metric space.

Huang and Zhang [8] also proved the following fixed point theorem in cone metric spaces.

Theorem 1.1. [8] Let $(X, d)$ be a complete cone metric space, $P$ be a normal cone with normal constant $K$. Suppose the mapping $T: X \rightarrow X$ satisfies the contractive condition

$$
d(T x, T y) \leq k d(x, y),
$$

for all $x, y \in X$ where $k \in[0,1)$ is a constant. Then $T$ has a unique fixed point in $X$ and for any $x \in X$, an iterative sequence $\left\{T^{n} x\right\}$ converges to the fixed point.

Eshaghi and et.al. [3] introduced the notion of orthogonal sets as follows (also see $[11,1,4,5,6,10])$ :

Definition 1.6. [3] Let $X \neq \phi$ and $\perp \subseteq X \times X$ be a binary relation. If $\perp$ satisfies the following condition

$$
\exists x_{0} ;\left(\left(\forall y ; y \perp x_{0}\right) \quad \text { or } \quad\left(\forall y ; x_{0} \perp y\right)\right),
$$

it is called an orthogonal set (briefly $O$-set). We denote this $O$-set by $(X, \perp)$.

Definition 1.7. Let $(X, \perp)$ be an O-set. A sequence $\left\{x_{n}\right\}_{n \in \mathbb{N}}$ is called an orthogonal sequence (briefly $O$-sequence) if

$$
\left(\left(\forall n ; x_{n} \perp x_{n+1}\right) \quad \text { or } \quad\left(\forall n ; x_{n+1} \perp x_{n}\right)\right) .
$$

for more information refer to [3].

Definition 1.8. [3] Let $(X, d, \perp)$ be an orthogonal metric space $((X, \perp)$ is an $O$ set and $(X, d)$ is a metric space). The space $X$ is orthogonally complete (briefly O-complete) if every Cauchy $O$-sequence is convergent.

It is easy to see that every complete metric space is O-complete and the converse is not true (see [3]).

Definition 1.9. [3] Let $(X, d, \perp)$ be an orthogonal metric space and $0<k<1$.

1. A mapping $f: X \rightarrow X$ is said to be orthogonal contractive ( $\perp$-contractive) mapping with Lipschitz constant $k$ if

$$
d(f x, f y) \leq k d(x, y) \quad \text { if } \quad x \perp y .
$$

2. A mapping $f: X \rightarrow X$ is called an orthogonal preserving $(\perp-$ preserving) mapping if $x \perp y$ then $f(x) \perp f(y)$. 
3. A mapping $f: X \rightarrow X$ is an orthogonal continuous ( $\perp$-continuous) mapping in $a \in X$ if for each $O$-sequence $\left\{a_{n}\right\}_{n \in \mathbb{N}}$ in $X$ if $a_{n} \rightarrow$ a then $f\left(a_{n}\right) \rightarrow f(a)$. Also $f$ is $\perp$-continuous on $X$ if $f$ is $\perp$-continuous in each $a \in X$.

They also, proved the following theorem which can be considered as a real extension of Banach fixed point theorem [11, 1, 3, 4, 5, 6, 10].

Theorem 1.2. [3] Let $(X, d, \perp$ ) be an O-complete metric space (not necessarily complete metric space). Let $f: X \rightarrow X$ be $\perp$-continuous, $\perp$-contraction (with Lipschitz constant $k$ ) and $\perp$-preserving, then $f$ has a unique fixed point $x^{*}$ in $X$. Also, $f$ is a Picard operator, that is, $\lim f^{n}(x)=x^{*}$ for all $x \in X$.

Let us consider the following periodic boundry value problem

$$
\left\{\begin{array}{l}
u^{\prime}(t)=f(t, u(t)), \\
u\left(t_{0}\right)=u(T),
\end{array}\right.
$$

where $t \in I=[0, T], T>0$ and $f: I \times \mathbb{R} \rightarrow \mathbb{R}$ is a continuous function. Suppose that there exists $\beta>0, \mu>0$ with $\mu<\beta$ such that for $x, y \in \mathbb{R}$ we have

$$
0 \leq|[f(t, y)+\beta y]-[f(t, x)+\beta x]| \leq \mu|y-x| .
$$

Inspired and motivated by the above results, we introduce new concept of orthogonal cone metric space. In such space, we establish new versions of fixed point theorems. As an application, we show the existence and uniqueness of solution of the periodic boundry value problem 1.1.

\section{Main Results}

In this section, we shall introduce a new definitions to prove the main results. We begin with the following definition. In the following part, we shall suppose $E$ is a Banach space, $P$ is a cone in $E$ with $i n t P \neq \phi$ and $\leq$ is partial ordering with respect to $P$.

Definition 2.1. Let $(X, \perp)$ be a nonempty orthogonal set. Suppose the mapping $d: X \times X \rightarrow E$ satisfies

- (d1) $0<d(x, y)$ for all $x, y \in X$ and $d(x, y)=0$ if and only if $x=y$;

- (d2) $d(x, y)=d(y, x)$ for all $x, y \in X$;

- (d3) $d(x, y) \leq d(x, z)+d(y, z)$ for all $x, y, z \in X$. 
Then $d$ is called a cone metric on $(X, \perp)$ and $(X, d, \perp)$ is called an orthogonal cone metric space.

We have the concept of orthogonal complete cone metric space as follows:

Definition 2.2. Let $(X, d, \perp)$ be an orthogonal cone metric space, if every Cauchy $O$-sequence is convergent in $X$, then $X$ is called an orthogonal complete cone metric space.

It is easy to see that every complete cone metric space is O-complete and the converse is not true. In the next example, $\mathrm{X}$ is $\mathrm{O}$-complete cone metric space and it is not complete.

Example 2.1. Let $E=\mathbb{R}, P=[0, \infty)$ and $X=[0,1)$. Suppose $x \perp y$ if $x \leq y .(X, \perp)$ is an O-set. Clearly, $X$ with metric $d: X \times X \rightarrow E$ such that $d(x, y)=|x-y|$ is not complete cone metric space but it is O-complete cone metric space. Because if $\left\{x_{k}\right\}$ is an arbitrary Cauchy O-sequence in $X$, then there exists a subsequence $\left\{x_{k_{n}}\right\}$ of $\left\{x_{k}\right\}$ for which $\left\{x_{k_{n}}\right\} \leq \frac{1}{2}$ for all $n$. It follows that $\left\{x_{k_{n}}\right\}$ converges to a $x \in\left[0, \frac{1}{2}\right] \subset X$. On the other hand, we know that every Cauchy sequence with a convergent subsequence is convergent. It follows that $\left\{x_{k}\right\}$ is convergent.

In the following example, we shall prove a theorem that can be considered as the main result of our paper.

Theorem 2.1. Let $(X, d, \perp$ ) be an orthogonal complete cone metric space (not necessarily complete cone metric space), $P$ be a normal cone with normal constant $K$. Suppose the mapping $T: X \rightarrow X$ is $\perp$-preserving, $\perp$-continuous and $\perp$-contraction Lipschitz constant $k \in[0,1)$. Then $T$ has a unique fixed point in $X$. In addition $T$ is a picard operator.

Proof. By definition of orthogonality, there exists $x_{0} \in X$ such that

$$
\left(\forall x \in X, x \perp x_{0}\right) \quad \text { or } \quad\left(\forall x \in X, x_{0} \perp x\right) .
$$

It follows that $x_{0} \perp T\left(x_{0}\right)$ or $T\left(x_{0}\right) \perp x_{0}$. Let

$$
x_{1}:=T\left(x_{0}\right), x_{2}:=T\left(x_{1}\right)=T^{2}\left(x_{0}\right), \cdots, x_{n+1}:=T x_{n}=T^{n}\left(x_{0}\right) .
$$

We have

$$
\begin{aligned}
d\left(x_{n+1}, x_{n}\right) & =d\left(T x_{n}, T x_{n-1}\right) \\
& \leq k d\left(x_{n}, x_{n-1}\right) \\
& \leq k^{2} d\left(x_{n-1}, x_{n-2}\right) \\
& \vdots \\
& \leq k^{n} d\left(x_{1}, x_{0}\right)
\end{aligned}
$$


So for $n>m$,

$$
\begin{aligned}
d\left(x_{n}, x_{m}\right) & \leq d\left(x_{n}, x_{n-1}\right)+d\left(x_{n-1}, x_{n-2}\right)+\cdots+d\left(x_{m-1}, x_{m}\right), \\
& \leq\left(k^{n-1}+k^{n-2}+\cdots+k^{m}\right) d\left(x_{1}, x_{0}\right), \\
& \leq \frac{k^{m}}{1-k} d\left(x_{1}, x_{0}\right) .
\end{aligned}
$$

We get $\left\|d\left(x_{n}, x_{m}\right)\right\| \leq \frac{k^{m}}{1-k} K\left\|d\left(x_{1}, x_{0}\right)\right\|$. This implies that $d\left(x_{n}, x_{m}\right) \rightarrow 0(n, m \rightarrow$ $\infty)$. Hence the O-sequence $\left\{x_{n}\right\}$ is Cauchy. By completeness of $X$, there exists $x^{*}$ in $X$ such that $x_{n} \rightarrow x^{*}(n \rightarrow \infty)$. On the other hand, $T$ is $\perp$-continuous and hence $T x_{n} \rightarrow T x^{*}$ as $n$ tends to infinity and $T\left(x^{*}\right)=\lim _{n \rightarrow \infty} T\left(x_{n}\right)=\lim _{n \rightarrow \infty} x_{n+1}=x^{*}$. Therefore $x^{*}$ is a fixed point of $T$.

To prove the uniqueness of the fixed point, let $y^{*} \in X$ be a fixed point of $T$. Then we have $T^{n}\left(y^{*}\right)=y^{*}$ for all $n \in \mathbb{N}$. By our choice of $x_{0}$ in the first part of the proof, we have

$$
x_{0} \perp y^{*} \quad \text { or } \quad y^{*} \perp x_{0} .
$$

Since $T$ is $\perp$-preserving, we have

$$
T^{n}\left(x_{0}\right) \perp T^{n}\left(y^{*}\right) \quad \text { or } \quad T^{n}\left(y^{*}\right) \perp T^{n}\left(x_{0}\right),
$$

for all $n \in \mathbb{N}$. On the other hand, $T$ is $\perp$-contraction, then we have for all $n \in \mathbb{N}$,

$$
\begin{aligned}
d\left(x^{*}, y^{*}\right) & =d\left(T^{n}\left(x^{*}\right), T^{n}\left(y^{*}\right)\right) \\
& \leq d\left(T^{n}\left(x^{*}\right), T^{n}\left(x_{0}\right)\right)+d\left(T^{n}\left(x_{0}\right), T^{n}\left(y^{*}\right)\right) \\
& \leq k^{n}\left[d\left(x^{*}, x_{0}\right)+d\left(x_{0}, y^{*}\right)\right] .
\end{aligned}
$$

Also we have

$$
\left\|d\left(x^{*}, y^{*}\right)\right\| \leq K\left(k^{n}\left[\left\|d\left(x^{*}, x_{0}\right)\right\|+\left\|d\left(x_{0}, y^{*}\right)\right\|\right]\right) .
$$

As $n$ goes to infinity, we get $x^{*}=y^{*}$.

Finally, we show that $T$ is a Picard operator. Let $x \in X$ be arbitrary. Similarly, then

$$
\left[x_{0} \perp x^{*} \text { and } x_{0} \perp x\right] \text { or }\left[x^{*} \perp x_{0} \text { and } x \perp x_{0}\right] .
$$

Now, since $T$ is $\perp$-preserving, then

$$
\left[T^{n}\left(x_{0}\right) \perp T^{n}\left(x^{*}\right) \text { and } T^{n}\left(x_{0}\right) \perp T(x)\right] \text { or }\left[T^{n}\left(x^{*}\right) \perp T^{n}\left(x_{0}\right) \text { and } T(x) \perp T^{n}\left(x_{0}\right)\right] \text {, }
$$

for all $n \in \mathbb{N}$. Hence for all $n \in \mathbb{N}$, we get

$$
d\left(T^{n}(x), T^{n}\left(x_{0}\right)\right) \leq k d\left(T^{n-1}(x), T^{n-1}\left(x_{0}\right)\right) \leq \cdots \leq k^{n} d\left(x, x_{0}\right) .
$$

Letting $n \rightarrow \infty$ we have $\lim _{n \rightarrow \infty} T^{n}(x)=x^{*}$. This completes the proof.

Here, we obtain another fixed point theorem by replacing $\perp$-contractive condition by another slightly modified condition. 
Theorem 2.2. Let $(X, d, \perp)$ be an orthogonal complete cone metric space, $P$ be a normal cone with normal constant $K$. Let $T: X \rightarrow X$ be $\perp$-preserving, $\perp$ continuous mapping satisfying the following $\perp$-contractive condition

$$
d(T x, T y) \leq a(d(x, y))+b[d(x, T x)+d(y, T y)]+c[d(x, T y)+d(y, T x)],
$$

for $x, y \in X$ with $x \perp y$ and the constants $a, b, c \in[0,1)$ and $a+b+c<1$. Then $T$ has a unique fixed point in $X$.

Proof. By definition of orthogonality, there exists $x_{0} \in X$ such that

$$
\left(\forall x \in X, x \perp x_{0}\right) \text { or }\left(\forall x \in X, x_{0} \perp x\right) .
$$

It follows that $x_{0} \perp T\left(x_{0}\right)$ or $T\left(x_{0}\right) \perp x_{0}$. Let

$$
x_{1}:=T\left(x_{0}\right), x_{2}:=T\left(x_{1}\right)=T^{2}\left(x_{0}\right), \cdots, x_{n+1}:=T x_{n}=T^{n}\left(x_{0}\right) .
$$

We have

$$
\begin{aligned}
& d\left(x_{n+1}, x_{n}\right)=d\left(T x_{n}, T x_{n-1}\right) \\
& \leq a\left(d\left(x_{n}, x_{n-1}\right)\right)+b\left[d\left(x_{n}, T x_{n}\right)+d\left(x_{n-1}, T x_{n-1}\right)\right]+c\left[d\left(x_{n}, T x_{n-1}\right)+d\left(x_{n-1}, T x_{n}\right)\right] \\
& \leq a\left(d\left(x_{n}, x_{n-1}\right)\right)+b\left[d\left(x_{n}, x_{n+1}\right)+d\left(x_{n-1}, x_{n}\right)\right]+c\left[d\left(x_{n}, x_{n}\right)+d\left(x_{n-1}, x_{n+1}\right)\right] \\
& \leq a\left(d\left(x_{n}, x_{n-1}\right)\right)+b\left[d\left(x_{n}, x_{n+1}\right)+d\left(x_{n-1}, x_{n}\right)\right]+c\left[d\left(x_{n-1}, x_{n}\right)+d\left(x_{n}, x_{n+1}\right)\right] .
\end{aligned}
$$

Therefore,

$$
d\left(x_{n+1}, x_{n}\right)(1-b-c)=d\left(x_{n}, x_{n-1}\right)(a+b+c),
$$

and we get

$$
d\left(x_{n+1}, x_{n}\right) \leq d\left(x_{n}, x_{n-1}\right) \frac{a+b+c}{1-b-c} .
$$

Substituting $k=\frac{a+b+c}{1-b-c}$ and as $0 \leq k<1$ we have

$$
d\left(x_{n+1}, x_{n}\right) \leq k d\left(x_{n}, x_{n-1}\right) \leq \cdots \leq k^{n} d\left(x_{1}, x_{0}\right) .
$$

For any $m \geq 1, p \geq 1$, it follows that

$$
\begin{aligned}
d\left(x_{m+p}, x_{m}\right) & \leq d\left(x_{m+p}, x_{m+p-1}\right)+d\left(x_{m+p-1}, x_{m}\right) \\
& \leq d\left(x_{m+p}, x_{m+p-1}\right)+d\left(x_{m+p-1}, x_{m+p-2}\right)+d\left(x_{m+p-2}, x_{m}\right) \\
& \leq d\left(x_{m+p}, x_{m+p-1}\right)+d\left(x_{m+p-1}, x_{m+p-2}\right)+d\left(x_{m+p-2}, x_{m+p-3}\right) \\
& +\cdots+d\left(x_{m+2}, x_{m+1}\right)+d\left(x_{m+1}, x_{m}\right) \\
& \leq k^{m+p-1} d\left(x_{1}, x_{0}\right)+k^{m+p-2} d\left(x_{1}, x_{0}\right)+k^{m+p-3} d\left(x_{1}, x_{0}\right) \\
& +\cdots+k^{m} d\left(x_{1}, x_{0}\right) \\
& \leq\left(k^{m+p-1}+k^{m+p-2}+k^{m+p-3}+\cdots+k^{m}\right) d\left(x_{1}, x_{0}\right) \\
& \leq \frac{k^{m}}{1-k} d\left(x_{1}, x_{0}\right)
\end{aligned}
$$


So we have

$$
\left\|d\left(x_{m+p}, x_{m}\right)\right\| \leq K \frac{k^{m}}{1-k}\left\|d\left(x_{1}, x_{0}\right)\right\| .
$$

Letting $m \longrightarrow \infty$ we conclude that $\left\{x_{n}\right\}$ is a Cauchy O-sequence. Since $(X, d)$ is a complete orthogonal cone metric space, there exists $x^{*} \in X$ such that $x_{n} \rightarrow x^{*}$ as $n \longrightarrow \infty$.

Next, we claim that $x^{*}$ is a fixed point of $T$.

$$
\begin{aligned}
d\left(T x^{*}, x^{*}\right) & \leq d\left(T x^{*}, T x_{n}\right)+d\left(T x_{n}, x^{*}\right) \\
& \leq d\left(T x^{*}, T x_{n}\right)+d\left(x_{n+1}, x^{*}\right),
\end{aligned}
$$

and we have

$$
\begin{aligned}
d\left(T x^{*}, x^{*}\right) & \leq a\left(d\left(x^{*}, x_{n}\right)\right)+b\left[d\left(x^{*}, T x^{*}\right)+d\left(x_{n}, T x_{n}\right)\right] \\
& +c\left[d\left(x^{*}, T x_{n}\right)+d\left(x_{n}, T x^{*}\right)\right]+d\left(x_{n+1}, x^{*}\right) \\
& \leq a\left(\left(d\left(x^{*}, x_{n}\right)\right)+b\left[d\left(x^{*}, T x^{*}\right)+d\left(x_{n}, x_{n+1}\right)\right]\right. \\
& +c\left[d\left(x^{*}, x_{n+1}\right)+d\left(x_{n}, T x^{*}\right)\right]+d\left(x_{n+1}, x^{*}\right) \\
& \leq a\left(\left(d\left(x^{*}, x_{n}\right)\right)+b\left[d\left(x^{*}, T x^{*}\right)+d\left(x_{n}, x^{*}\right)+d\left(x^{*}, x_{n+1}\right)\right]\right. \\
& +c\left[d\left(x^{*}, x_{n+1}\right)+d\left(x_{n}, x^{*}\right)+d\left(x^{*}, T x^{*}\right)\right]+d\left(x_{n+1}, x^{*}\right) .
\end{aligned}
$$

So

$$
d\left(T x^{*}, x^{*}\right)(1-b-c) \leq d\left(x^{*}, x_{n}\right)(a+b+c)+d\left(x^{*}, x_{n+1}\right)(1+b+c),
$$

and

$$
d\left(T x^{*}, x^{*}\right) \leq \frac{d\left(x^{*}, x_{n}\right)(a+b+c)+d\left(x^{*}, x_{n+1}\right)(1+b+c)}{(1-b-c)} .
$$

Therefore

$$
\left\|d\left(T x^{*}, x^{*}\right)\right\| \leq K\left(\frac{(a+b+c)}{(1-b-c)}\left\|d\left(x^{*}, x_{n}\right)\right\|+\frac{(1+b+c)}{(1-b-c)}\left\|d\left(x^{*}, x_{n+1}\right)\right\|\right) .
$$

Letting $n \longrightarrow \infty$, we have $T x^{*}=x^{*}$.

Finally, we need to prove that the fixed point is unique.

If there is another fixed point $y^{*}$, then

$$
\begin{aligned}
d\left(x^{*}, y^{*}\right) & =d\left(T x^{*}, T y^{*}\right) \\
& \leq a\left(d\left(x^{*}, y^{*}\right)\right)+b\left[d\left(x^{*}, T x^{*}\right)+d\left(y^{*}, T y^{*}\right)\right]+c\left[d\left(x^{*}, T y^{*}\right)+d\left(y^{*}, T x^{*}\right)\right] \\
& \leq a\left(d\left(x^{*}, y^{*}\right)\right)+b\left[d\left(x^{*}, T x^{*}\right)+d\left(y^{*}, T y^{*}\right)\right] \\
& +c\left[d\left(x^{*}, T x^{*}\right)+d\left(T x^{*}, T y^{*}\right)+d\left(y^{*}, T y^{*}\right)+d\left(T y^{*}, T x^{*}\right)\right] \\
& =(a+2 c) d\left(x^{*}, y^{*}\right) .
\end{aligned}
$$

$$
(1-a-2 c) d\left(x^{*}, y^{*}\right) \leq 0
$$

this implies that

$$
\left\|d\left(x^{*}, y^{*}\right)\right\|=0 .
$$

Hence $x^{*}=y^{*}$. Therefore the proof is completed. 


\section{Application in differential equations}

In this section, we apply results in the previous section to show the existence and uniqueness of solution of the following periodic boundary value problem 1.1 where $t \in I=[0, T], T>0$ and $f: I \times \mathbb{R} \rightarrow \mathbb{R}$ is a continuous function. Let

$X=\{u \in C(I, \mathbb{R}) ; u(t)>1 \quad\{$ for almost every $\} \quad t \in I\}$. Consider the Banach space $E=\mathbb{R}$ and $P=[0, \infty)$. Define partial ordering $\leq$ with respect to $P$ by $a \leq b$ if and only if $b-a \in P$.

Suppose the mapping $d: X \times X \rightarrow E$ by

$$
d(x, y)=\sup _{t \in I}|x(t)-y(t)|
$$

for $x, y \in X$.

Suppose that there exists $\beta>0, \mu>0$ with $\mu<\beta$ such that for $x, y \in \mathbb{R}$ we have 1.2 .

Theorem 3.1. Under above conditions, for all $T>0$ the differential equation 1.1 has a unique solution.

Proof. The problem can be written in integral equation as

$$
x(t)=\int_{0}^{T} G(t, s)[f(s, x(s))+\beta x(s)] d s
$$

where

$$
G(t, s)= \begin{cases}\frac{e^{\beta(T+s-t)}}{e^{\beta T}-1}, & 0 \leq s \leq t \leq T \\ \frac{e^{\beta(s-t)}}{e^{\beta T}-1}, & 0 \leq t \leq s \leq T\end{cases}
$$

Define the following orthogonality relation $\perp$ in $X$ :

$$
x \perp y \quad \text { if } \quad x(t) y(t) \geq y(t)
$$

for almost every $t \in I$. It's easy to see that $(X, d, \perp)$ is a cone metric space. Since every $x$ is a continuous function over a closed and bounded subset of the Euclidean space, this supremum is actually attained in $(X, d, \perp)$. Hence $(X, d, \perp)$ is complete.

Now, we define $A:(X, d, \perp) \rightarrow(X, d, \perp)$ as follows:

$$
(A x)(t)=\int_{0}^{T} G(t, s)[f(s, x(s))+\beta x(s)] d s,
$$

for all $t \in I$.

Note that the fixed points of $A$ are the solutions of 1.1. 
First, we claim that for every $x \in X, A x \in X$. To see this, for every $t \in I$ and $x \in X$, we have

$$
\begin{aligned}
A x(t) & =\int_{0}^{T} G(t, s)[f(s, x(s))+\beta x(s)] d s \\
& =\int_{0}^{T} G(t, s) f(s, x(s)) d s+\int_{0}^{T} G(t, s) \beta x(s) d s \\
& >\int_{0}^{T} G(t, s) f(s, x(s)) d s+\beta \int_{0}^{T} G(t, s) d s \\
& \left.\left.=\int_{0}^{T} G(t, s) f(s, x(s)) d s+\beta \frac{1}{e^{\beta T}-1}\left(\frac{1}{\beta} e^{\beta(T+s-t)}\right]_{0}^{t}+\frac{1}{\beta} e^{\beta(s-t)}\right]_{t}^{T}\right) \\
& =\int_{0}^{T} G(t, s) f(s, x(s)) d s+\beta \frac{1}{\beta} \\
& =\int_{0}^{T} G(t, s) f(s, x(s)) d s+1 .
\end{aligned}
$$

one can conclude that $A x(t)>1$ and we have $A x \in X$.

Now, we check that the hypotheses in Theorem 2.1 is satisfied. To this end, we prove the following statements:

1. $A$ is $\perp$-preserving,

2. $A$ is $\perp$-contraction,

3. $A$ is $\perp$-continuous

1. We recall that $A$ is $\perp$-preserving if for every $x, y \in X, x \perp y$ we have $A x \perp A y$. We have shown above that $A x(t)>1$ for all $t \in I$, which implies that $A x(t) A y(t) \geq A y(t)$ for all $t \in I$. So $A x \perp A y$ if $x \perp y$.

2. Let $x, y \in X$ and $x \perp y$, we have

$$
\begin{aligned}
|A x(t)-A y(t)| & =\left|\int_{0}^{T} G(t, s)[f(s, x(s))+\beta x(s)-f(s, y(s))-\beta y(s)] d s\right| \\
& \leq \int_{0}^{T}|G(t, s)||\mu(x(t)-y(t))| d s \\
& \leq \mu|x(t)-y(t)| \int_{0}^{T} G(t, s) d s \\
& =\frac{\mu}{\beta}|x(t)-y(t)| .
\end{aligned}
$$

So,

$$
d(A x, A y)=\sup _{t \in I}|A x(t)-A y(t)| \leq \frac{\mu}{\beta} \sup _{t \in I}|x(t)-y(t)|=\frac{\mu}{\beta} d(x, y) .
$$


The inequality 3.2 shows that $A$ is $\perp$-contraction with Lipschitz constant $\lambda=\frac{\mu}{\beta}<1$.

3. Let $\left\{x_{n}\right\}$ be an O-sequence in $X$ such that $\left\{x_{n}\right\}$ converges to some $x \in X$. Since $A$ is $\perp$-preserving, $\left\{A x_{n}\right\}$ is an O-sequence. For each $n \in \mathbb{N}$, by (2), we have

$$
\left|A x_{n}-A x\right| \leq \lambda\left|x_{n}-x\right| .
$$

As $n$ tends to infinity, it follows that $A$ is $\perp$-continuous.

The mapping $A$ defined above satisfies the hypotheses of the Theorem 2.1. Thus, the existence and uniqueness of its fixed point $x^{*} \in X$ has been guaranteed by Theorem 2.1. As we noted above, $x^{*}$ is a unique solution to differential equation 1.1 .

\section{REFEREN CES}

1. H. Baghani, M. Eshaghi Gordji and M. Ramezani, Orthogonal sets: their relation to the axiom of choice and a generalized fixed point theorem, J. Fixed Point Theory Appl. (2016) 18: 465.

2. W.S. Du, A note on cone metric fixed point theory and its equivalence, Nonlinear Anal. 72 (2010) 2259-2261.

3. M. Eshaghi Gordji, M. Ramezani, M. De La Sen and Y. J. Cho, On orthogonal sets and Banach fixed point theorem, Fixed Point Theory. 18 (2017) no. 2, 569-578.

4. M. Eshaghi and H. Habibi, Fixed point theory in generalized orthogonal metric space, J. Linear Topol. Algebr. 06 (2017) no. 03, 251-260.

5. M. Eshaghi, H. Habibi and M. B. Sahabi, Orthogonal sets; orthogonal contractions, Asian-European J. Math. Accepted.

6. M. Eshaghi and H. Habibi, Existence and uniqueness of solutions to a first-order differential equation via fixed point theorem in orthogonal metric space, FACTA UNIVERSITATIS (NIS) Ser. Math. Inform. Accepted.

7. S. Jankovic, Z. Kadelburg and S. Radenvic, On cone metric spaces: A survey, Nonlinear Analysis. 74 (2011) 2591-2601.

8. L.G. Huang and X. Zhang, Cone metric spaces and fixed point theorems of contractive mappings, J. Math. Anal. Appl. 332 (2007) 1468 -1476.

9. J. R. Morales and E. Rojas, Cone metric spaces and fixed point theorems of Tcontractive mappings, Revista Notas de Matematica. vol.4(2), no. 269 (2008) 66-78.

10. M. Ramezani and H. Baghani, The Mier-Keeler fixed point theorem in incomplete modular spaces with application, J. Fixed Point Theory Appl. (2017), Volume 19, Issue 4, 2369-2382.

11. A. Bahraini, G. Askari, M. Eshaghi Gordji and R. Gholami, Stability and hyperstability of orthogonally *-m-homomorphisms in orthogonally Lie $C^{*}$-algebras: a fixed point approach, J. Fixed Point Theory Appl. (2018), 20:89. 
Zeinab Eivazi Damirchi Darsi Olia

Department of Mathematics

Islamic Azad University

South Tehran Branch, Tehran, Iran

St_z_eivazi@azad.ac.ir

Madjid Eshaghi Gordji

Department of Mathematics

Semnan University

P.O. Box 35195-363

Semnan, Iran

meshaghi@semnan.ac.ir

Davood Ebrahimi Bagha

Department of Mathematics

Islamic Azad University

Central Tehran Branch, Tehran, Iran

E_bagha@yahoo.com 